\title{
Benzodiazepine Use in Breast Cancer Survivors: Findings from a Consecutive Series of 1,000 Patients
}

\author{
Rakhee Vaidya $^{\mathrm{a}}$ Richa Sood $^{\mathrm{a}}$ Nina Karlin $^{\mathrm{c}}$ Aminah Jatoi ${ }^{\mathrm{b}}$

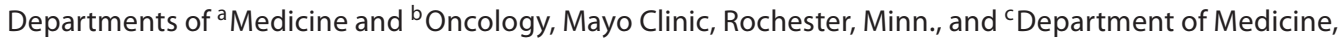 \\ Mayo Clinic, Scottsdale, Ariz., USA
}

Key Words

Anxiety $\cdot$ Cancer survivors $\cdot$ Benzodiazepine $\cdot$ Breast cancer

\begin{abstract}
Objective: This study reports the percentage of breast cancer survivors receiving ongoing benzodiazepines and the circumstances surrounding their usage. Methods: The medical records of 1,000 consecutive breast cancer survivors who were no longer receiving adjuvant chemotherapy were reviewed. Results: Among those patients, 7.9\% (95\% confidence interval 6.2-9.6; higher than the 3\% rate in the general population) were receiving benzodiazepines. Lorazepam was most commonly prescribed. Sixty-eight patients were cancer free at their last visit, and 51 had not been taking benzodiazepines prior to their cancer diagnosis. Anxiety was the single most frequent reason for initiating and continuing benzodiazepines. Conclusion: Anxiety appears to be a common explanation for relatively high rates of benzodiazepine use in breast cancer survivors. This finding merits further study.

Copyright $\odot 2011$ S. Karger AG, Base
\end{abstract}

Prescribed for decades for anxiety disorders, benzodiazepines have now been replaced by more appropriate interventions such as selective serotonin reuptake inhibi- tors along with cognitive behavioral therapy $[1,2]$. The potential for abuse, lack of antidepressant properties, risk of addiction, and harrowing withdrawal all spawn concerns over the long-term use of benzodiazepines. Yet, benzodiazepines continue to be used on an ongoing basis in as many as $3 \%$ of adults in the general population [3].

What percentage of cancer survivors continue to use benzodiazepines even after completion of most of their cancer therapy? This question is relevant for at least two reasons. First, over one third of cancer patients and cancer survivors report anxiety [4]. Although, to our knowledge, previous studies have not evaluated rates of and reasons for ongoing benzodiazepine use among cancer survivors, this anxiety suggests a need to explore these issues. Second, recent guidelines from the American Society of Clinical Oncology and other organizations include benzodiazepines within their antiemetic armamentarium [5]. Because state-of-the-art adjuvant breast cancer chemotherapy is highly emetogenic, benzodiazepines are frequently prescribed adjunctively, thus underscoring the importance of investigating ongoing benzodiazepine use in breast cancer survivors.

Hence, the goals of the current study were to report the percentage of breast cancer survivors receiving benzodiazepine prescriptions and to describe the types of benzodiazepines prescribed and the circumstances surrounding their use.

\section{KARGER}

두 2011 S. Karger AG, Basel

Fax +41613061234 E-Mail karger@karger.ch www.karger.com www.karger.com/ocl
Aminah Jatoi, MD

Mayo Clinic

200 First Street SW

Rochester, MN 55905 (USA)

Tel. +1 507284 3902, E-Mail jatoi.aminah@mayo.edu 


\section{Methods}

Overview and Patient Selection

The Mayo Clinic Institutional Review Board approved this study. The Mayo Clinic Tumor Registry aided in obtaining medical records.

This study focused on patients consecutively seen at the Mayo Clinic in Rochester, Minn., USA, immediately prior to 2009. Patients who were seen from within any specialty or subspecialty were assessed. Patients with even a remote diagnosis of breast cancer were included; cancer diagnosis or treatment need not have occurred at the Mayo Clinic. The above criteria reflect an intention to assess current practice patterns, exclude patients on adjuvant chemotherapy and garner a robust estimate of benzodiazepine use in breast cancer survivors.

\section{Data Acquisition}

One thousand consecutive medical records were screened for benzodiazepine prescriptions. The medical records of patients who had been prescribed benzodiazepines were then reviewed in depth by one of the authors (R.V.). These prescriptions were not necessarily written by Mayo Clinic healthcare providers and may have been prescribed by others. No distinction was made based on whether prescriptions were written with as-needed or aroundthe-clock directions of usage.

The latter yielded information on patient age at the last documented recording of benzodiazepine use, when benzodiazepine was first prescribed, the date of diagnosis of breast cancer, whether or not chemotherapy had been administered, the patient's cancer status at the time of benzodiazepine documentation, marital status at the last benzodiazepine prescription, psychiatric illness (as descriptively presented in the medical record and regardless of whether formal psychiatric evaluation had been pursued), and the vital status at the time of record review. Information was also gathered on the type of benzodiazepine prescribed, the date of the last benzodiazepine prescription, and the reason for the first/ last prescription. If specific dates were not available, the investigators estimated with a mid-year or mid-month date.

\section{Sample Size Calculation}

One thousand patients enabled reporting the percentage of benzodiazepine use with $95 \%$ confidence intervals within $3 \%$ of the most conservative estimates.

\section{Results}

\section{Benzodiazepine Prescriptions and Patient \\ Demographics}

Among 1,000 breast cancer survivors, 7.9\% were prescribed benzodiazepines (95\% confidence interval 6.29.6).

The median age among these patients was 79 years (range 52-105). Other demographics are listed in table 1. Sixty-one remain alive at the time of this report. Sixtyeight were cancer free at their last clinic visit, 9 had recurrent cancer, and in 2, the cancer status was unknown.
Table 1. Demographics at the last benzodiazepine prescription

$\begin{array}{lc}\text { Age, years } & \\ \text { Median } & 79 \\ \text { Range } & 52-105 \\ \text { Marital status } & \\ \text { Married } & 36(46) \\ \text { Widowed } & 29(37) \\ \text { Divorced } & 9(11) \\ \text { Single } & 5(6) \\ \text { Ethnicity } & \\ \text { White } & 78(99) \\ \text { Unknown } & 1(1) \\ \text { Chemotherapy given for breast cancer } & \\ \text { Yes } & 12(15) \\ \text { No } & 51(65) \\ \text { Unknown } & 16(20) \\ \text { Cancer status } & \\ \text { Cancer free } & 68(86) \\ \text { Recurrence } & 9(11) \\ \text { Unknown } & 2(3) \\ \text { Underlying psychiatric illness } & \\ \text { Depression/anxiety } & \\ \text { Adjustment disorder/anxiety } & 76(33) \\ \text { Panic disorder } & 7(9) \\ \text { Personality disorder } & 2(3) \\ \text { Not specified } & 1(1) \\ & 1(1)\end{array}$

Figures in parentheses are percentages.

\section{Benzodiazepines and Reasons for Prescriptions}

The median interval from breast cancer diagnosis to last benzodiazepine prescription was 14 years (range 1-51). Fifty-one patients were not taking benzodiazepines prior to their cancer diagnosis, and only 12 were. This information is unknown in the other patients. Information on when the first benzodiazepine prescription was given was not consistently available in the medical record.

The following benzodiazepines were prescribed: lorazepam $(n=43)$, diazepam $(n=13)$, alprazolam $(n=10)$, clonazepam $(\mathrm{n}=10)$, oxazepam $(\mathrm{n}=1)$, temazepam $(\mathrm{n}=$ $1)$, and triazolam $(n=1)$. These benzodiazepines were first prescribed for one or more of the following reasons: anxiety $(\mathrm{n}=34)$, insomnia $(\mathrm{n}=12)$, muscle spasms $(\mathrm{n}=$ $6)$, restlessness $(n=5)$, dyspnea $(n=3)$, nausea $(n=2)$, tremor $(n=1)$, seizures $(n=1)$, depression $(n=1)$, or unknown $(n=24)$. They were continued for one or more of the following reasons: anxiety $(n=51)$, insomnia $(n=10)$, nausea $(n=6)$, muscle spasms $(n=6)$, restlessness $(n=6)$, shortness of breath $(n=1)$, essential tremor $(n=1)$, seizures $(n=1)$, or unknown $(n=13)$, with 14 patients having 
more than one indication for continued use of benzodiazepines. Thirty-nine of these patients on benzodiazepines were taking other anxiolytics and/or antidepressants.

\section{Discussion}

This cross-sectional study of benzodiazepine use found that $7.9 \%$ (95\% confidence interval 6.2-9.6) of breast cancer survivors were prescribed these drugs, despite the fact that best patient practice patterns show that the anxiety-related indication for these agents has waned. Even after confining this report to breast cancer survivors with no recurrent cancer or to those who started taking benzodiazepines only after their cancer diagnosis, this percentage still exceeds the $3 \%$ benchmark in the general population and sits on the outer limits of other comparative data $[3,6,7]$. Admittedly, the rate of general benzodiazepine use can be variable, depending on the clinical and geographic characteristics of the assessed patient population, but nonetheless, the rate reported here seems higher than expected [8]. We emphasize the point that our methodology allowed us to detect only the percentage of patients prescribed this class of medication and not confirmed rates of usage.

Moreover, we had expected to see higher rates of benzodiazepine use among patients who had received adjuvant chemotherapy because of their need for antiemetics. However, many current benzodiazepine users had not received adjuvant chemotherapy. Importantly, a substantial number of patients were taking these agents specifically for anxiety. Apparently, patients who were diagnosed with breast cancer many years earlier and who remain cancer free may still be struggling with the psychological ramifications of their malignant diagnosis. Our results suggest that healthcare providers should probe into anx- iety-related symptoms among breast cancer survivors and consider prescribing better, more effective treatments, such as selective serotonin reuptake inhibitors and cognitive behavioral therapy.

Another important point centers on recent data that show that certain drugs inhibit the metabolism of tamoxifen by CYP2D6 and thereby potentially lessen the efficacy of the latter. Although benzodiazepines have not been strongly implicated in this manner, these high rates of continued anxiolytic use underscore the importance of continued vigilance for drug interactions among cancer survivors who may over time be prescribed a variety of drugs for anxiety and other mood disorders.

Our findings should be viewed with caution. First, this study examined patients who were seeking medical care in a tertiary medical center. Breast cancer survivors followed at non-tertiary facilities may have fewer medical problems, less anxiety, and hence, lower rates of benzodiazepine use. Second, the retrospective nature of this study is such that we are unable to account for why all these patients were first prescribed benzodiazepines or to describe details surrounding their breast cancer treatment. A survey-based assessment in breast cancer survivors might more precisely elucidate the reasons behind these prescribing patterns.

In conclusion, the current study observed a high rate of benzodiazepine use among breast cancer survivors. These findings should be confirmed in other settings, invite further study to explain them, probe into their consequences, and caution healthcare providers to remain sensitive to anxiety in breast cancer survivors.

\section{Acknowledgment}

This work was supported by K24CA131099.

\section{References}

1 Coben JH, Davis SM, Furbee PM, et al: Hospitalizations for poisoning by prescription opioids, sedatives, and tranquilizers. Am J Prev Med 2010;38:517-524.

2 Cloos J-M, Ferreira V: Current use of benzodiazepines in anxiety disorders. Curr Opin Psychiatry 2008;22:90-95.

-3 Esposito E, Barbui C, Patten SB: Patterns of benzodiazepine use in a Canadian population. Epidemiol Psychiatr Soc 2009;18:248254

Benzodiazepines in Cancer Survivors
4 Mosher CE, Duhamel KN: An examination of distress, sleep, and fatigue in metastatic breast cancer patients. Psychooncology 2010, Epub ahead of print.

5 Kris MG, Hesketh PJ, Somerfield MR, et al: American Society of Clinical Oncology guideline for antiemetics in oncology: update 2006. J Clin Oncol 2006;24:2932-2947.

-6 Lagnaoui R, Depont F, Fourrier A, et al: Patterns and correlates of benzodiazepine use in the French general population. Eur J Clin Pharmacol 2004;60:523-529.
7 Fang S-Y, Chen C-Y, Chang I-S, et al: Predictors of the incidence and discontinuation of long-term use of benzodiazepines: a population-based study. Drug Alcohol Depend 2009;104:140-146.

8 Centers for Disease Control and Prevention: Emergency department visits involving nonmedical use of selected prescription drugs. United States 2004-2008. MMWR Morb Moral Wkly Rep 2010;59:705-709. 